\title{
MOVIMIENTO HACIA EL REFUERZO DE LA PROTECCIÓN PATENTARIA DE LOS MEDICAMENTOS EN ADPIC PLUS ¿VERSUS O A FAVOR DEL ACCESO A LA SALUD? ${ }^{1}$
}

\author{
MOVEMENT TOWARDS THE REINFORCEMENT OF PATENTARY \\ PROTECTION OF DRUGS IN TRIPS PLUS: AGAINST OR IN FAVOR \\ OF ACCESS TO HEALTH?
}

\section{MOVIMENTO PARA O REFORÇO DA PROTEÇÃO PATENTÁRIA DOS MEDICAMENTOS NO TRIPS PLUS: CONTRA OU A FAVOR DO ACESSO À SAÚDE?}

\section{MARTA CAROLINA GIMÉNEZ PEREIRA}

Doctora en Derecho por el Instituto de Investigaciones Jurídicas de la Universidad Nacional Autónoma de Mexico (IIJ UNAM). Posdoctora en Derecho por la Facultad Meridional (IMED), Río Grande do Sul, Brasil. Profesora Investigadora Visitante, Programa de Posgrado en Derecho PPGD de la Universidad Federal de Bahía (UFBA), Brasil. Líder del Grupo de Pesquisa/Seminario Permanente en Propiedad Intelectual y Nuevas Tecnologías PINTEC del Posgrado en Derecho PPGD-UFBA y miembro del Grupo de Estudios en Desarrollo, Innovación y Propiedad Intelectual GEDIPI del Posgrado en Derecho IMED. Líneas de investigación: Propiedad Intelectual y Nuevas Tecnologías, Biotecnología, Bioética. E-mail: magipe@hotmail.com.

\footnotetext{
1 Texto basado en conferencia presentada por la autora en el VII Seminario Internacional "Democracia e efetividade da jurisdição nacional e internacional" del Programa de Posgrado en Derecho de la Universidad de Itaúna, Minas Gerais, el 6 de junio de 2019.
} 


\section{RESUMEN}

Las nuevas tendencias en propiedad intelectual nos remiten al primigenio ADPIC para el análisis necesario de temáticas de derecho a la competencia y propiedad intelectual en el sector farmacéutico y más tarde arribar a las implicancias del futuro de ADPIC, a saber, ADPIC PLUS, con la polémica específica de la "información no divulgada" y, en ella, la protección de la "exclusividad de datos" que ya se encontraba prevista en el artículo 39.3 de aquél. Empero, existen difíciles interpretaciones para esta debatida figura que no escapa a los intereses de los distintos sectores participantes de los variados países miembros de la OMC. El presente estudio abarca dos temáticas diferenciadas que se desarrollan después de la introducción, la cual tiene por objeto colocar al lector en contexto. En una primera parte, se aborda la relación de la propiedad intelectual con el derecho de la competencia, generando luego dos propuestas anticompetitivas. En la segunda parte, aparece la figura de la exclusividad de datos como parte integrante de las nuances de extensión patentaria gestadas en el escenario internacional y las estrategias resultantes, desde sus conceptos y requisitos, analizando sus interpretaciones, así como las sensibilidades en su aplicación. El método utilizado es el deductivo y la técnica de investigación bibliográfica. Se estructura en introducción, desarrollo, conclusiones y bibliografía.

PALABRAS-CLAVE: Patentes farmacéuticas; Innovación; Propiedad Intelectual; Acceso a la salud; Derecho de la competencia; Acuerdo ADPIC Plus; Artículo 39.3 ADPIC.

\section{ABSTRACT}

The new trends in intellectual property refer us to the original TRIPS agreement for the necessary analysis on topics in competition law and intellectual property in the pharmaceutical sector and later arrive at the implications of the future of TRIPS, that is, TRIPS PLUS, with the specific controversy of the "undisclosed information" and, in it, the protection of "data exclusivity" that was already foreseen in the former's article 
39.3. However, there are difficult interpretations for this debated figure that do not escape the interests of different participating sectors in the various countries that are members of the WTO. The present study covers two differentiated themes that are scrutinized after the introduction, which aims to put the reader in context. At the first part, relations between intellectual property and competition law are addressed, thus generating two anticompetitive proposals. At the second part, the figure of data exclusivity appears as an integrating part of the patented extension nuances conceived on international stage and the resulting strategies, from their concepts and requirements, analyzing their interpretations, as well as the sensitivities in their application. The method used is the deductive and the bibliographic research technique. It is structured in introduction, development, conclusions and bibliography.

KEYWORDS: Pharmaceutical patents; Innovation; Intellectual property; Access to health; Competition law; TRIPS PLUS Agreement; Article 39.3 TRIPS

\section{RESUMO}

As novas tendências na propriedade intelectual nos remetem ao primordial Acordo TRIPS para a necessária análise de temáticas do direito da concorrência e propriedade intelectual no setor farmacêutico e, mais tarde para se chegar às implicações do futuro do TRIPS, a saber, o TRIPS PLUS, com a específica polêmica da "informação não divulgada" e, nesta, sobre a proteção da "exclusividade de dados" que já se encontrava prevista no art. 39.3 daquele primeiro. No entanto, existem interpretações difíceis para esta figura debatida que não escapa aos interesses dos diferentes setores participantes dos vários países membros da OMC. O presente estudo abarca duas temáticas diferenciadas que se desenvolvem após a introdução, a qual tem por objetivo colocar o leitor em contexto. Na primeira parte, aborda-se a relação da propriedade intelectual com o direito da concorrência, gerando então duas propostas anticoncorrenciais. Na segunda parte, aparece a figura da exclusividade de dados como parte integrante das nuances de extensão patentária gestadas no cenário 
internacional e as estratégias resultantes, desde seus conceitos e requisitos, analisando suas interpretações, bem como as sensibilidades em sua aplicação. O método utilizado é o dedutivo e a técnica de investigação bibliográfica. Se estrutura em introdução, desenvolvimento, conclusões e bibliografia.

PALAVRAS-CHAVE: Patentes farmacêuticas; Inovação; Propriedade intelectual; Acesso à saúde; Direito da concorrência; Acordo TRIPS PLUS; Artigo 39.3 TRIPS.

\section{INTRODUCCIÓN}

A priori se constata que, más allá de que la intención de los gobiernos haya sido la de impulsar sus sectores nacionales en el rubro de la innovación con la promulgación de leyes de propiedad intelectual adecuadas a la normativa internacional y un abanico de programas de fomento, la política pública aún cede espacio ante la presencia acaparadora de grupos farmacéuticos trasnacionales. Estos agentes económicos, mayormente extranjeros, dotados de un mayor potencial de inversión en innovación, han crecido de forma significante en el mercado nacional siendo detentores de posición dominante. En tal sentido, se analiza que el accionar de modo general de los países en vías de desarrollo para la protección de los derechos de propiedad intelectual, ya incluía una adopción de estrategias desde el primigenio Acuerdo sobre los Aspectos de los Derechos de Propiedad Intelectual relacionados con el Comercio (en adelante, ADPIC o TRIPS, por sus siglas en inglés).

En la intención de hacer frente al comportamiento anticompetitivo, se identifican asimetrías profundas entre los países pobres y los ricos en cuanto al incentivo al desarrollo y comercialización de medicamentos lucrativos, lo que ha provocado el surgimiento de medidas propuestas por la Organización Mundial de la Salud (en adelante OMS) para fomentar la innovación a través de un listado de mecanismos alternativos desarrollados en conjunto con asociaciones de capital público y privado. 
Como propuestas anticompetitivas, en este trabajo se analizan dos temáticas de propiedad intelectual y derecho de la competencia relevantes para el mercado farmacéutico: la competencia desleal y acceso a la tecnología y la "negativa a tratar" de la licencia voluntaria como presupuesto de la licencia obligatoria.

Por otro lado, nuevas tendencias en propiedad intelectual nos remiten al ADPIC al analizar la "información no divulgada" y en ella la "exclusividad de datos" prevista en su artículo 39.3. Empero, la debatida figura conlleva distantes interpretaciones según los ojos de los intereses de los distintos sectores y países presentes en la Organización Mundial de Comercio (en adelante $\mathrm{OMC}$ ). El trabajo describe de tal forma las implicancias del futuro de ADPIC, a saber, ADPIC PLUS, en la exclusividad de datos.

La metodología utilizada es deductiva y de revisión bibliográfica, a partir de la cual serán destacadas informaciones técnicas e históricas con tendencia analítica y didáctica.

\section{EL ACUERDO ADPIC Y LAS PROFUNDAS ASIMETRÍAS DE SUS PAÍSES FIRMANTES EN SUS DERECHOS DE PROPIEDAD INTELECTUAL Y EN EL ACCESO A LOS MEDICAMENTOS}

La intención de este apartado es analizar y entender los intereses de los países en vías de desarrollo en la observancia de los derechos de propiedad intelectual en su vínculo con el derecho de la competencia y el cuidado del interés público, abordando la ofensiva que pretendida para los países menos desarrollados.

Se ha debatido largamente acerca de estrategias genuinas de cara al Acuerdo ADPIC, que conforman los emergentes ADPIC PLUS, como un conjunto de acciones tendientes a atacar los derechos de propiedad intelectual, propiciados por los países en vías de desarrollo.

En ADPIC se destaca, de hecho, el artículo 40 que dispone la posibilidad de incluir estipulaciones sobre prácticas restrictivas específicamente en los "contratos de 
licencia" como punto de observancia para garantizar el balance adecuado entre la competencia y la protección de los derechos de propiedad intelectual.

Tal artículo forma parte de la Parte II del Acuerdo, Sección 8 denominada "Control de las prácticas anticompetitivas en las licencias contractuales", dentro de las "Normas relativas a la existencia, alcance y ejercicio de los derechos de Propiedad Intelectual", donde se establece a la letra:

1. Los Miembros convienen en que ciertas prácticas o condiciones relativas a la concesión de las licencias de los derechos de propiedad intelectual, que restringen la competencia, pueden tener efectos perjudiciales para el comercio y pueden impedir la transferencia y la divulgación de la tecnología. 2. Ninguna disposición del presente Acuerdo impedirá que los Miembros especifiquen en su legislación las prácticas o condiciones relativas a la concesión de licencias que puedan constituir en determinados casos un abuso de los derechos de propiedad intelectual que tenga un efecto negativo sobre la competencia en el mercado correspondiente. Como se establece supra, un Miembro podrá adoptar, de forma compatible con las restantes disposiciones del presente Acuerdo, medidas apropiadas para impedir 0 controlar dichas prácticas, que pueden incluir las condiciones exclusivas de retrocesión, las condiciones que impidan la impugnación de la validez y las licencias conjuntas obligatorias, a la luz de las leyes y reglamentos pertinentes de ese Miembro. 3. Cada uno de los Miembros celebrará consultas, previa solicitud, con cualquiera otro Miembro que tenga motivos para considerar que un titular de derechos de propiedad intelectual que es nacional del Miembro al que se ha dirigido la solicitud de consultas o tiene su domicilio en él realiza prácticas que infringen las leyes o reglamentos del Miembro solicitante relativos a la materia de la presente sección, y desee conseguir que esa legislación se cumpla, sin perjuicio de las acciones que uno y otro Miembro pueda entablar al amparo de la legislación ni de su plena libertad para adoptar una decisión definitiva. El Miembro a quien se haya dirigido la solicitud examinará con toda comprensión la posibilidad de celebrar las consultas, brindará oportunidades adecuadas para la celebración de las mismas con el Miembro solicitante y cooperará facilitando la información públicamente disponible y no confidencial que sea pertinente para la cuestión de que se trate, así como otras informaciones de que disponga el Miembro, con arreglo a la ley nacional y a reserva de que se concluyan acuerdos mutuamente satisfactorios sobre la protección de su carácter confidencial por el Miembro solicitante. 4. A todo Miembro cuyos nacionales o personas que tienen en él su domicilio sean en otro Miembro objeto de un procedimiento relacionado con una supuesta infracción de las leyes o reglamentos de este otro Miembro relativos a la materia de la presente Sección este otro Miembro dará, previa petición, la posibilidad de celebrar consultas en condiciones idénticas a las previstas en el párrafo 3 (OMC, 1994).

\section{En las patentes de productos farmacéuticos las patentes cumplen una función} reconocida de financiar las actividades de investigación y desarrollo $(\mathrm{I}+\mathrm{D})$ en la 
industria y también sus proyectos. Las patentes sólo funcionan ante mercados redituables y esto es una consecuencia lógica de su propia naturaleza: ellas brindan un incentivo que sólo puede concretarse cuando existen las condiciones adecuadas en términos de recursos humanos, capital y rentabilidad prevista (CORREIRA, 2009, p.93).

A pesar de las expectativas que originaron los nuevos rumbos de la $I+D$ en los países en vías de desarrollo, como en el caso de la India, resulta evidente que las compañías multinacionales aún se enfocan en desarrollar nuevos fármacos que interesan a los mercados ricos. El modelo adoptado por las compañías de la India ha sido el de desarrollar nuevas moléculas para luego, todavía en su etapa incipiente de desarrollo, cederlas a través de licencias a grandes compañías farmacéuticas evitando así aquellas tener que solventar elevados costos de los ensayos clínicos y sus posteriores aprobaciones por la autoridad sanitaria local que, por si fuera poco, además demoran bastante tiempo hasta llegar a su etapa final (CHAUDHURI, 2005). Si bien las instituciones públicas de I+D han constituido la principal fuente de descubrimientos de uso eventualmente farmacéutico, es la industria farmacéutica quien en su mayor parte ha financiado y llevado a cabo la fase de desarrollo de los productos farmacéuticos, incluso esos costosos y prolongados ensayos clínicos.

Es que el papel que juega la industria farmacéutica es crucial: sigue siendo uno de los sectores más rentables de la economía: cuarta posición luego del de minería, producción de petróleo crudo y bancos comerciales (WHO, 2006)2 ${ }^{2}$, según informe de la Comisión en Derechos de Propiedad Intelectual, Innovación y Salud Pública (CIPIH en adelante) del año 2006 que incluso revela que la industria farmacéutica fue el sector más redituable desde los años 80 hasta el año 2003.

Recordemos por otro lado aquel período de transición concedido por ADPIC ${ }^{3}$ para los países de desarrollo, países menos adelantados y economías en transición

\footnotetext{
${ }^{2}$ Cfr. Comission on Intellectual Property Rights, Innovation and Public Heath (CIPIH), informe titulado "Public Health, Innovation and Intellectual Property Rights", World Health Organization, año 2006, edición en internet.

${ }^{3}$ Que obligó a los países signatarios a establecer un período uniforme mínimo de 20 años para la protección por patente, modificando así sus legislaciones internas existentes. Entre tales países también se encontraban Canadá y Estados Unidos.
} 
que no reconocían previamente a tal Acuerdo la protección de los productos farmacéuticos por patente y que tuvieron hasta el 1 de enero de 2005 para poder implementar progresivamente en sus legislaciones y en la práctica administrativa las novedades de la norma internacional: sólo unos pocos países pudieron utilizar plenamente tal flexibilidad.

Las patentes, a pesar del establecimiento de regímenes sui generis tendientes a una mayor participación de diversos actores intervinientes en la extensa cadena de $I+D$, siguen mostrando que contribuyen al desarrollo de nuevos tratamientos sólo cuando existen mercados redituables de magnitud. En consecuencia, la falta de demanda en el tratamiento de enfermedades de los países pobres hace que la protección por patentes sea irrelevante para enfermedades denominadas de tipo III, y sólo relativamente importantes para las del tipo II.

La conclusión en este punto es que las patentes pueden instrumentalizar, así presentadas, las profundas desigualdades entre países pobres y ricos al incentivar el desarrollo y comercialización de fármacos redituables y no de aquellos que se necesitan con toda urgencia a fin de tratar problemas de salud que aquejan a la mayor parte de la población mundial. Todo ello devela la importancia de desarrollar mecanismos alternativos para promover la innovación farmacéutica no sólo en ciertos países y sus sectores más desarrollados, sino por sobre todo en aquellos alternativos y que involucran enfermedades que padece la población pobre, lo que será abordado en el siguiente apartado.

\section{LA PROPUESTA DE LA OMS PARA PROMOVER LA INNOVACIÓN: MECANISMOS ALTERNATIVOS ANTE NECESIDADES DE SALUD URGENTES}

Como respuesta al planteamiento, la Organización Mundial de la Salud (OMS en adelante) junto con varias asociaciones de capitales públicos y privados para desarrollar productos considerados necesarios en países en vías de desarrollo, a pesar de las serias dudas sobre la sustentabilidad de dichas iniciativas, ha iniciado un 
debate sobre creación e implementación de mecanismos alternativos para promover la innovación, que incluía un posible tratado internacional en la materia ${ }^{4}$. Se propusieron los siguientes mecanismos alternativos:

- Exclusividad en el mercado por tiempo limitado basada en el modelo de "medicamentos huérfanos" aplicado en los Estados Unidos bajo la Ley de Medicamentos Huérfanos de 1983. Recibe la crítica de que a pesar de haber funcionado aparentemente en tal país (CIPIH, 2019, p.70-77), parece funcionar sólo cuando el poder adquisitivo es alto y la exclusividad es criticada por negar el acceso a nuevos fármacos a los pacientes con menos ingresos (CORREA, 2009, p.95).

- Recompensas en forma de premios para el desarrollo de medicamentos para enfermedades que prevalecen en países en vías de desarrollo: útil en el incentivo de desarrollar nuevas drogas y que no sean sometidas al régimen de patentes ${ }^{5}$.

- Compromisos de compra por adelantado: donde se garantice la compra a futuro de ciertas cantidades de producto a ser desarrollado y por un precio acordado, incluso con un mecanismo de reducción de precios luego de cierto plazo de tiempo: este mecanismo funciona cuando ya se ha identificado una molécula y el riesgo en la $1+D$ es relativamente bajo.

- Proyectos de "acceso abierto" en particular para la identificación de moléculas nuevas potenciales: el modelo puede albergar avances en las primeras etapas del ciclo de I+D de productos farmacéuticos lo cual fue abordado por la llamada Agenda para el Desarrollo de la OMPI ${ }^{6}$.

\footnotetext{
${ }^{4}$ El Grupo de Trabajo sobre Innovación, PI y Salud Pública se reunió por primera vez en diciembre de 2005.

5 Joseph Stiglitz, premio Nóbel de economía 2001, proponía en 2007 la creación de un fondo para premios para el desarrollo de nuevos productos. Ver en Times Online MBA Podcast in association with IBM: Week 1 - Making Globalisation Work.

${ }^{6}$ La cual fue propuesta por Argentina y Brasil en 2004. El Comité Provisional sobre Propuestas relacionadas con la Agenda de la OMPI para el Desarrollo (PCDA) tenía en 2009 unas 111 propuestas a considerar y que han sido presentadas en la sesión celebrada en 2006 y que han sido agrupadas en diversos grupos.
} 
- Un nuevo tratado internacional sobre investigación médica, propuesto por un número de ONGs y gobiernos que asegure el financiamiento sostenible de la I+D de productos farmacéuticos (CORREA, 2009, p.95-97).

Un ejemplo de éxito de estos mecanismos es el nacimiento de una asociación entre la Iniciativa Fármacos para las Enfermedades Desatendidas (DNDi) y el laboratorio Sanofi Aventis que en conjunto desarrolló una combinación de dosis fija de artesunate-amodiaquina (ASAQ), que se presentó para su registro en 23 países africanos del Sub-Sahara y que en el año 2009 se registró en 12 de esos países ${ }^{7}$.

\section{PROPIEDAD INTELECTUAL Y DERECHO DE LA COMPETENCIA. UN FRENTE AL COMPORTAMIENTO ANTICOMPETITIVO: DOS TEMATICAS RELEVANTES}

En su vínculo con el derecho de la competencia, el derecho que protege las invenciones necesita analizar la pertinencia de enfrentar actitudes anticompetitivas, lo que será expuesto como dos propuestas que se enuncian a continuación.

\subsection{COMPETENCIA DESLEAL Y ACCESO A LA TECNOLOGÍA}

El mercado de tecnología merece atención separada del mercado de bienes y servicios y también sufre de conductas anticompetitivas. "Cuando se comercializan derechos de propiedad intelectual, independientemente de los productos a los que corresponden, también debe definirse el mercado tecnológico de referencia. Los mercados de tecnologías incluyen los derechos de propiedad intelectual que se conceden bajo licencia, así como las tecnologías alternativas..." la Comisión ya indicaban en ese entonces que la tecnología es un insumo que se

\footnotetext{
7 Véase noticias en DNDi.

8 Cfr. las antiguas "Directrices sobre la aplicabilidad del artículo 81 del Tratado de la CE a los acuerdos de cooperación horizontal (2001/C 3/02)", disponible en el Boletín Oficial de la Unión Europea del $6 / 1 / 2001$. Este texto ya no está vigente y fue sustituido el 14 de enero de 2011 por el denominado 52011 XC0114 (04), que se intitula "Directrices sobre la aplicabilidad del artículo 101 del Tratado de Funcionamiento de la Unión Europea a los acuerdos de cooperación horizontal, Texto pertinente a efectos del EEE", disponible en edición en internet en: <https://eur-lex.europa.eu/legalcontent/es/AUTO/?uri=CELEX:52011XC0114(04)>.
} 
integra en un producto o en un proceso de producción y, por tanto, las licencias que involucran tecnología pueden afectar la competencia.

Los mercados de tecnología consisten en propiedad intelectual que ha sido licenciada (tecnología licenciada) y sus sustitutos cercanos. Cuando los derechos de propiedad intelectual se comercializan de manera separada de los productos en los cuales se utilizan, los Organismos pueden basarse en los mercados de tecnología para analizar los efectos competitivos de un acuerdo de licenciamiento9.

La intención de la política en materia de competencia aborda los casos en que la propiedad intelectual (que en adelante la llamaremos PI) es utilizada para aplicar precios excesivos sobre las tecnologías protegidas o para impedir el acceso a ellas (CORREA, 2009, p.10).

Correa, en tal sentido, enfatiza que los derechos de PI no son absolutos ya que están sujetos a intereses públicos más elevados y no implica entonces que gocen de una inmunidad frente a la intervención del derecho de la competencia. Debe entenderse que los mismos se otorgan para servir los intereses públicos a través de los inventores y creadores, no sólo para beneficiarlos, según un análisis utilitario hecho a diferencia de una conceptualización efectuada desde el punto de vista del derecho natural de propiedad. Lo fundamental no es discutir sobre si la política de competencia puede interferir con tales de derechos de PI sino sobre cuándo se justifica tal intervención (CORREA, 2009, p.10-11).

La cuestión de los precios excesivos aplicados sobre las tecnologías protegidas o la restricción del acceso a ellas a través de diversas estrategias anticompetitivas debe ser atendida como un derecho primordial del consumidor. El punto más sensible se presenta en los países en vías de desarrollo donde, como consecuencia última, se resiente la innovación local con muy pocos incentivos, agregando sólo efectos positivos en la inversión extranjera que es la que resulta beneficiada en última instancia en estos países, monopolizando o impidiendo además el correcto andamiento de innovaciones subsiguientes.

${ }^{9}$ Cfr. Directrices sobre Defensa de la Competencia para la Concesión de Licencias de Propiedad Intelectual del Departamento de Justicia y la Comisión Federal de Comercio del 4/6/1995. 
Estamos frente a un caso de política pública sectorial de Estado que no está sujeta al calendario de administración de gobierno.

\subsection{NEGATIVA A TRATAR COMO PRESUPUESTO DE LAS LICENCIAS OBLIGATORIAS}

Esta situación se relaciona con la dispensación de autorización para la utilización de la materia protegida por el titular del derecho de PI a un tercero. Es decir, la posibilidad de prescindir del consentimiento del detentor del derecho a estos efectos. Tal hipótesis aparece típicamente al relacionar estos derechos con el de la competencia.

Hurgando en el concepto de derecho de propiedad, se pensaría que se hace indispensable la autorización, a priori. Las circunstancias que habilitarían tal excepción tienen que ver generalmente con la necesidad de entrar a competir en mercados monopolizados o los casos de falta de explotación de patentes, emergencias o intereses públicos que habilitan la celebración de las llamadas licencias obligatorias.

La "negativa a tratar" se refiere a una acción unilateral de no otorgar una licencia voluntaria sobre una patente y se basa en el mismo Acuerdo ADPIC, según su artículo $31 b^{10}$.

Ahora bien, si bien la disposición sólo se refiere a la "negativa a otorgar" una licencia voluntaria como condición para emitir una obligatoria, la Secretaría de la OMC ha reconocido expresamente la posibilidad de articular una "negativa a tratar" como

\footnotetext{
10 Tal artículo 31 de ADPIC dispone: "Otros usos sin autorización del titular de los derechos. Cuando la legislación de un Miembro permita otros usos de la materia de una patente sin autorización del titular de los derechos, incluido el uso por el gobierno o por terceros autorizados por el gobierno, se observarán las siguientes disposiciones: ... b) sólo podrán permitirse esos usos cuando, antes de hacerlos, el potencial usuario haya intentado obtener la autorización del titular de los derechos en términos y condiciones comerciales razonables y esos intentos no hayan surtido efecto en un plazo prudencial. Los Miembros podrán eximir de esta obligación en caso de emergencia nacional o en otras circunstancias de extrema urgencia, o en los casos de uso público no comercial. Sin embargo, en las situaciones de emergencia nacional o en otras circunstancias de extrema urgencia el titular de los derechos será notificado en cuanto sea razonablemente posible. En el caso de uso público no comercial, cuando el gobierno o el contratista, sin hacer una búsqueda de patentes, sepa o tenga motivos demostrables para saber que una patente válida es o será utilizada por o para el gobierno, se informará sin demora al titular de los derechos".
} 
motivo suficiente para emitir dichas licencias, lo cual se ha contemplado en varias legislaciones nacionales, como las de China, Argentina y Alemania (CORREA, 2009, p.16). En el caso argentino, la Ley de Patentes establece que será otorgada una licencia obligatoria si el titular de la patente no concede la licencia voluntaria dentro de un período de 150 días a partir del pedido efectuado por el tercero, bajo términos y condiciones comerciales razonables ${ }^{11}$.

\section{IMPLICANCIAS DE ADPIC PLUS EN LA FIGURA DE LA EXCLUSIVIDAD DE DATOS. EL ARTÍCULO 39.3 DE ADPIC}

Los procesos de adhesión a acuerdos bilaterales, multilaterales, de libre comercio, con tendencia en alza y que comporta obligaciones para las partes que son muchas veces irrenunciables a pesar de la discrecionalidad que prevé igualmente el ADPIC a la hora de adoptar la norma, hacen olvidar a los sujetos negociadores y a los titulares de derechos aspectos imprescindibles de considerar, tales como el hecho de que el uso de datos por parte del gobierno no importaría un uso comercial desleal, que existen casos que escapan a la figura por tratarse de utilidad pública o de emergencia sanitaria previstas en la ley a fin de controlar el monopolio en casos específicos (licencias obligatorias), que el costo de la exclusividad de datos en países en vías de desarrollo no puede ser absorbido por los laboratorios de producción local, así como cuestiones referentes a las actuales tendencias que negocian en secrecía los países industrializados.

\footnotetext{
${ }^{11}$ Ley de Patentes argentina: "CAPITULO VII. OTROS USOS SIN AUTORIZACION DEL TITULAR DE LA PATENTE. ARTICULO 42: Cuando un potencial usuario haya intentado obtener la concesión de una licencia del titular de una patente en términos y condiciones comerciales razonables en los términos del artículo 43 y tales intentos no hayan surtido efecto luego de transcurrido un plazo de CIENTO CINCUENTA (150) días corridos contados desde la fecha en que se solicitó la respectiva licencia, el INSTITUTO NACIONAL DE LA PROPIEDAD INDUSTRIAL, podrá permitir otros usos de esa patente sin autorización de su titular. Sin perjuicio de lo mencionado precedentemente, se deberá dar comunicación a las autoridades creadas por la Ley N 22.262 o la que la modifique o sustituya, que tutela la libre concurrencia a los efectos que correspondiere", disponible en MINISTERIO DE JUSTICIA Y DERECHOS HUMANOS. PRESIDENCIA DE LA NACIÓN, 1996).
} 


\subsection{CONCEPTUALIZACION, NORMATIVIDAD INTERNACIONAL E INTERPRETACIÓN DE LA PROTECCIÓN DE DATOS DE PRUEBA: EL INICIO DE LA POLÉMICA}

Es el artículo 39.3 del ADPIC de 1994, el que introduce la regulación internacional, de forma vinculante, sobre lo que se conoce como "información no divulgada" y la protección de los datos de prueba sobre la seguridad y eficacia de productos farmacéuticos y agroquímicos. Veamos primeramente qué comprende.

CORREA nos dice que según ADPIC la noción cubre dos categorías diferentes de información: la que se conoce como "secretos comerciales" y la que nos ocupa, que son los "datos de prueba". Los datos de prueba se definen como los resultados de ensayos clínicos que se llevan a cabo para demostrar la seguridad y eficacia de productos farmacéuticos y agroquímicos y según el autor su protección fue unos de los temas que consiguió dividir el norte y el sur durante las negociaciones de la Ronda Uruguay, en su momento (CORREA, 2014, p.13-14).

La protección consiste en la prohibición de utilización de tales datos para obtener la aprobación de comercialización de un producto farmacéutico o agroquímico. Consiste en un derecho de protección que ostenta el titular de los datos contra prácticas comerciales desleales, evitándose expresamente el uso o la divulgación, salvo casos específicos. Conlleva ese derecho una obligación de no hacer, obligación negativa o de abstención, a más de una obligación de protección consecuente.

Se trata de una prerrogativa otorgada por los países miembros de la Organización Mundial de Comercio, en adelante OMC, que obliga a proteger los datos de prueba llevados a cabo por el titular contra prácticas de competencia desleal, siendo esta disciplina la que regula la figura de la información no divulgada expresada más arriba, según surge del artículo 10 bis del Convenio de París ${ }^{12}$.

12 Este artículo 10 bis del Convenio de París dispone: "Competencia desleal: 1) Los países de la Unión
están obligados a asegurar a los nacionales de los países de la Unión una protección eficaz contra la
competencia desleal. 2) Constituye acto de competencia desleal todo acto de competencia contrario a
los usos honestos en materia industrial o comercial. 3) En particular deberán prohibirse: i) cualquier
acto capaz de crear una confusión, por cualquier medio que sea, respecto del establecimiento, los 
Diferentes interpretaciones se han dado a raíz de esta redacción del artículo 39.3, tal como en la práctica ocurrió con diversos acuerdos bilaterales y multilaterales celebrados entre países miembros de la OMC. El principal argumento en contra de esta protección la encuentran una vez más las patentes de medicamentos y los detractores se sostienen con fuerza en los efectos que la norma acarrea señalando que si el gobierno utiliza los datos de prueba para aprobar versiones genéricas de medicamentos no existe uso desleal ${ }^{13}$ y añaden que tal disposición de ADPIC no debería conllevar en automático una concesión de exclusividad ni remuneración.

Un sensible punto se presenta sobre estos matices de esos efectos: la intervención del gobierno en la entrada de los medicamentos genéricos como parte de sus políticas públicas, así como el impedimento de que la normativa citada de ADPIC se constituyen en una suerte de trampolín para acceder a mayores derechos que los que confiere una patente, tentando beneficiarse de exclusividades y regalías bajo el argumento de la debatida figura de "data protection", como se conoce en inglés.

Estados Unidos y la Unión Europea argumentan que el mencionado artículo 39.3 de ADPIC ordena la protección de datos y su exclusividad para el titular. Sin embargo, el sentido ordinario de la terminología empleada en ese artículo, interpretado en el contexto de la terminología de "competencia desleal" en el Convenio de París y más aún en la negociación histórica del artículo 39.3, pone en duda este punto de vista (BASHEER, 2006, p.11).

La polémica del siempre pretender más de una norma o ir más allá de la norma es esa espina que hinca, desatada por Estados Unidos ya en aquel entonces, que no ha encontrado un punto de detenimiento y que se refleja incluso en el denominado ADPIC PLUS así como en el Acuerdo Transpacífico o TPP por su siglas en inglés, a la fecha de hoy no muerto sino latente a pesar de la postura política norteamericana

productos o la actividad industrial o comercial de un competidor; ii) las aseveraciones falsas, en el ejercicio del comercio, capaces de desacreditar el establecimiento, los productos o la actividad industrial o comercial de un competidor; iii) las indicaciones o aseveraciones cuyo empleo, en el ejercicio del comercio, pudieren inducir al público a error sobre la naturaleza, el modo de fabricación, las características, la aptitud en el empleo o la cantidad de los productos".

${ }^{13} \mathrm{El}$ uso de tales datos por parte del gobierno a través de sus entidades a fin de estudiar aplicaciones subsiguientes con miras a que obtengan la aprobación de comercialización no puede considerarse como un "uso comercial". 
de 2017 que parecía acabar con él, análisis que no corresponde a este estudio pero que resulta pertinente mencionar a modo ilustrativo.

Otra de las problemáticas que rondan en cuanto a la interpretación del artículo en comento se refiere a qué tipo de protección deben dar los países partes de la OMC a esta figura, es decir, al alcance de la norma a la hora de celebrar los Tratados de Libre Comercio (en adelante TLCs).

Por un lado, la doctrina ha estudiado literalmente el artículo 39.3 y de la opinión académica se colige que bastaría con circunscribir la figura a la disciplina de la competencia desleal, la cual no exige el reconocimiento ni la aplicación de derechos exclusivos, añadiendo que más bien el otorgamiento de la exclusividad constituiría una derogación drástica del principio de libre competencia ${ }^{14}$. De hecho, el ADPIC contiene suficientes flexibilidades para que un país determine los medios adecuados para proteger los datos de prueba ${ }^{15}$. El mismo párrafo 4 de la Declaración de Doha señala que las disposiciones "... deben ser interpretadas y aplicadas de manera tal que se avale el derecho de los Miembros de la OMC de proteger la salud pública y, en particular, de promover el acceso a los medicamentos para todos" y por lo tanto debería adoptarse la postura de usarse las flexibilidades de ADPIC teniendo en cuenta el interés nacional del país de que se trate (SATWANT, 2007, p.4), lo cual parece ser lo más equilibrado.

Por otro lado, si la norma internacional establece la obligación de exclusividad, deja a los países miembros la tarea de determinar la duración de tal obligación con el consecuente riesgo de que alguno de ellos otorgue tal protección por un tiempo ilimitado a su titular lo que vendría a acentuar la brecha entre los países industrializados y aquellos en vías de desarrollo en cuanto a la discusión de la

\footnotetext{
${ }^{14}$ En tal sentido autores como Carlos Correa, Charles Clift, Lucas Arrivillaga, Jean-Frédéric Morin, Jerome Reichman, Pedro Roffe, Christoph Spennemann, citados por Correa.

${ }^{15}$ De acuerdo con el Acuerdo ADPIC, si la autoridad regulatoria de un país solicita la presentación de datos no divulgados al titular para el registro del medicamento o agroquímico, la exigencia para el país miembro de la OMC no es sino la de proteger esos datos contra su uso comercial desleal en caso de tratarse de una nueva entidad química y en caso de que la elaboración de tales datos haya supuesto un "esfuerzo considerable". Más adelante se apuntará la postura que el concepto abarcaría el de "uso comercial" de forma amplia y no tan sólo el "uso comercial desleal".
} 
pertinencia de la protección o inclusive entre aquellos o éstos mismos en lo que respecta a la duración del período de exclusividad.

Hay que tener el cuidado en apuntar que los países en desarrollo han sentado la postura en este sentido, negándose en su mayoría a conceder la protección ${ }^{16}$ : el artículo 39.3 ADPIC deja un espacio considerable a los países miembros para implementar la obligación de proteger datos de prueba; la información no divulgada se encuentra ya regulada por la competencia desleal en el artículo 10 bis del Convenio de París; la información no divulgada no debe ser abordada dentro del concepto de "propiedad" y no exige conceder derechos exclusivos al titular de los datos ${ }^{17}$.

Los detractores de la protección en comento han sustentado su razonamiento en que la investigación y desarrollo $(I+D)$ de nuevos productos farmacéuticos y agroquímicos es impulsada fundamentalmente por países desarrollados con lo cual la figura de exclusividad de datos no debería aplicarse en países en desarrollo por la razón de que siendo que los desarrollados representan el $85 \%$ del mercado mundial de medicamentos bajo receta de productos farmacéuticos, es improbable que la falta de esa exclusividad en uno o más países en desarrollo sea determinante para una empresa a la hora de decidir invertir en producir los datos de prueba. Por otro lado, sostiene que el tiempo de ventaja que obtiene la compañía que origina tales datos, así como la misma figura de la patente como vía de protección, resultan suficientes para recuperar los costos del desarrollo de tales datos (CORREA, 2014, p.20-21).

Sin embargo, esto es discutible porque la intención primigenia de la norma es precisamente la de ofrecer oportunidades comerciales más justas a los titulares de datos y sobre todo armonizar un diseño de políticas efectivas con legítimos reclamos de derechos exclusivos limitados por el interés público y que se puedan defender en

\footnotetext{
16 Tendencia que en los últimos años se vio disminuida en razón de la presión que ejerce la OMC, la firma de un TLC inclusive por presiones unilaterales. Entre los países que la han rechazado se citan Argentina e India. En el caso de Colombia, al haber suscrito diversos TLCs con Estados Unidos, la Asociación Europea de Libre Comercio (AELC) y con la Unión Europea, se suma al compromiso de otorgar protección a los datos de prueba concediendo períodos de exclusividad. Canadá es otro de los países que se cita entre los que han otorgan el período de exclusividad de datos. Inclusive lo han ampliado de cinco a ocho años, más seis meses de exclusividad pediátrica, según las enmiendas del año 2006 hechas al Reglamento Canadiense sobre Alimentos y Fármacos.

17 Postura sentada en reunión del Consejo del Acuerdo sobre los ADPIC.
} 
términos del bienestar público real ${ }^{18}$, promoviendo la necesidad de aumentar las actividades de innovación e I+D en productos farmacéuticos también en países en vías de desarrollo o aquellos que son productores de principios activos de productos farmacéuticos y agroquímicos, como el caso de India, en forma adecuada y utilizando tanto el rico capital humano existente a nivel local como su infraestructura ${ }^{19}$. En esta armonización se incluye el incentivo de lograr el progreso de los países en vías de desarrollo. El argumento de que hoy en día la mayoría de los productos farmacéuticos y agroquímicos son elaborados por países desarrollados, teniendo una posición de mercado dominante, se encontraría en cierta forma limitado precisamente por el reclamo de mayor presencia de $1+D$ en aquellos países, sin desdeñar la realidad propia que los incumbe y las propias diferencias entre tales países en vías de desarrollo. El ejemplo es la India como productor importante de principios activos.

Aun así, en las negociaciones del mencionado artículo 39.3, las partes en su mayoría rechazaron la propuesta norteamericana eligiendo más bien hacer hincapié a la aplicación de las reglas de competencia desleal el cual no implica el reconocimiento de derechos exclusivos. En la disputa de introducción de la exclusividad de datos, como se ha mencionado, sea a través de modificaciones a la legislación nacional, de negociaciones de TLCs o de acuerdos bilaterales, así como a través de los procesos de adhesión a la OMC de nuevos integrantes, se ha notado que la representación de la industria de productos de marca o big pharma ha apoyado activamente la introducción de cinco o más años de exclusividad en tanto que el segmento genérico de la industria farmacéutica ha disentido enérgicamente con esta postura (CORREA, 2014, p.22-23).

Según se anticipó, otros matices tienen que ver con la diferencia entre "basarse" y "utilizar" amén de que dependerá de quién sea quien los utiliza pues, como se ha visto, el gobierno, para cumplir su tarea a través de autoridades, precisa usar

\footnotetext{
18 Como expresa Antony Taubman, actual Director de la División de PI de la OMC, que propone una lectura heterodoxa del artículo 39.3, basándose en una interpretación de relación justa entre competidores y el cumplimiento de las expectativas de negociaciones de los países sobre el acceso a la protección de la PI.

${ }_{19}$ Cfr. op. cit., informe de la Declaración Ministerial de Doha en Doha WTO Ministerial 2001 del 20 noviembre 2001,
} 
esos datos a fin de evaluar aplicaciones futuras que procurarán la aprobación de la comercialización, lo cual no implica un uso "comercial" de tales datos. Además, hablando de una posible ventaja comercial que puedan tener los competidores con tal uso, ella es totalmente lícita si no conlleva prácticas deshonestas. Y es que, hasta en esta última palabra cabrían sutilezas si consideramos que lo "desleal" y lo "deshonesto" dependerá de la cultura que se analice.

Podemos concluir en este tópico que la obligación que tienen los países parte conlleva una protección con libertad de determinar la forma de proteger esos datos. La obligación supone:

1) No obtener datos de prueba no divulgados por medio de prácticas deshonestas.

2) No dar a los datos los datos de prueba un uso comercial.

\subsection{LA DOCTRINA DE LA JUSTA RETRIBUCIÓN}

Siempre en cuanto a la interpretación de la norma, pero añadiendo la justa retribución por el uso de los datos de prueba, autores sostienen que la figura puede ser admitida toda vez que se garantice una remuneración al titular por su uso, cumplidos los dos presupuestos básicos que son: que los datos de las entidades farmacéuticas o agroquímicas no hayan sido divulgados y que su producción implique un esfuerzo considerable.

Afirma BASHEER, al hacer el estudio del caso de la India, que el estándar mínimo que impone el artículo 39.3 no es la exclusividad de datos (postura de EEUU y de la UE) ni la denominada de dependencia permisiva (postura de algunos autores) sino m (ás bien un modelo de "responsabilidad compensatoria" por el cual la información reglamentaria que cumple con los requisitos previos del artículo 39.3 recientemente citados como presupuestos básicos- no puede ser utilizada por ninguna persona, como tampoco por autoridades regulatorias en su labor de aprobación de nuevos productos, sin que se remunere al titular de los datos (BASHEER, 2006, p.38). En sentido acorde se ha pronunciado FELLMETH apostando por una remuneración 
adecuada a fin de que la divulgación y el uso de los datos sean justos desde un punto de vista económico (FELLMETH, 2004). Y MEITINGER revela que el artículo 39.3 requiere que los países miembros garanticen el uso exclusivo de los datos durante un período determinado o que fijen un pago en contraprestación por tal uso (MEITINGER, 2005, p.125-137).

Hecha la exposición de la doctrina, expondré a continuación mis conclusiones sobre la temática en el apartado correspondiente y que sigue.

\section{CONCLUSIONES}

Respecto al crecimiento de las empresas extranjeras que se da por la tendencia continua en celebrar fusiones y adquisiciones de empresas nacionales de pequeño y mediano porte, esta situación revela una traba para el crecimiento de la industria nacional en el entendido de que la ventaja económica obtenida por las innovadoras foráneas se muestra en que la comercialización de los medicamentos no da un retorno a la economía interna, al menos como debería. El mercado farmacéutico actúa como un oligopolio en base lo dicho, caminando en la actualidad a una clara posición dominante en el mercado, lo cual amenaza tanto al público consumidor como a los competidores nacionales, otros detentores de oligopolios pero en menor medida.

La situación debe ser controlada a través de herramientas y medidas adecuadas que expone el moderno derecho de la competencia así como por otras medidas alternativas, tales como las propuestas por la OMS y que tienen un foco hacia situaciones urgentes de enfermedades específicas de zonas geográficas delimitadas, lo que debería ser implementado en el corto plazo.

Respecto a las implicancias de ADPIC PLUS en la figura de la exclusividad de datos, ellas dependerán de la interpretación de la norma que en las rondas negociadoras los países miembros de la OMC o firmantes de los respectivos TLCs o acuerdos bilaterales den a la figura. Uno de los mayores temores en la adopción de la figura está en el hecho de que ella acarrearía un aumento en el coste de las medicinas 
y productos agroquímicos, limitando el acceso a los mismos. El coste se vería aumentado en razón de la misma repetición y práctica en la obtención de los datos de prueba por los laboratorios genéricos, lo cual significa nuevos costos de todo tipo que, en caso de que éstas puedan afrontarlas, repercuten en el precio final del producto ya puesto a la venta. Así, el financiamiento de prolongados ensayos particularmente en países en desarrollo retrasa la actividad y el progreso científico amén de acrecentar el valor del medicamento o del agroquímico, en su caso. Hay que considerar además que la tecnología con la que cuentan y el personal que labora en tales compañías de producción de medicamentos genéricos suele ser limitado y no alcanza el nivel de infraestructura humana o científica ni la capacidad suficiente de soportar financieramente o solventar estudios prolongados, a veces en forma indefinida hasta alcanzar el resultado querido.

Otro, quizás el más sensible, aborda la temática de poner en riesgo la vida y la salud del paciente repitiendo innecesariamente pruebas ya existentes $\sin$ justificación científica. Por otro lado, el hecho de que cuánto tiempo puede esperar la sociedad para acceder a medicinas genéricas es una pregunta que también comporta un tinte ético donde se encuentra el factor imprescindible de contemplar el acceso a los medicamentos que debe convivir con el del fomento a la innovación de forma equitativa, mas no en detrimento suyo.

No hay que dejar de mencionar que, hasta la figura de las licencias obligatorias, tan necesarias como importantes, pueden verse sensibilizadas ante la presencia de la temática que nos ocupa pues en la negociación de TLCs es incierto si el titular de una licencia obligatoria que forma parte de un país firmante de tal tratado puede obtener aprobación para comercializar un producto en caso de que el mismo esté gozando de la protección de exclusividad de datos, salvo que el titular de éstos le conceda la respectiva autorización. Sin ahondar en este tema, cabría también preguntarnos la conveniencia de la actual tendencia en alza de alentar firmas de tratados multilaterales o los TLCs, ya que en la práctica lo que puede ocurrir en temas de acceso a la salud es que el gobierno signatario se vea constreñido a participar de políticas que no le son favorables, viéndose trabadas herramientas que han sido 
contempladas con éxito por los ADPIC a fin de medir o limitar los monopolios que otorgan los instrumentos de $\mathrm{PI}$, como sería el caso de estos permisos que en los países en desarrollo se convierten en la clave para asegurar el acceso a los medicamentos a pacientes que de otra manera no podrían contar con ellos. Resulta un poco contradictorio pensar que un paciente deba estar sujeto a permisos que deban concederse entre titulares: del titular de la licencia obligatoria del medicamento que quedaría supeditado al titular de los datos de prueba del mismo que ejerce una suerte de exclusividad de mercado.

Como se adelantó, hay que tener el cuidado en apuntar que el artículo 39.3 ADPIC deja un espacio considerable a los países miembros para implementar la obligación de proteger datos de prueba, que la información no divulgada se encuentra ya regulada por la competencia desleal en el artículo 10 bis del Convenio de París, así como que la información no divulgada no debe ser abordada dentro del concepto de "propiedad" y no exige conceder derechos exclusivos al titular de los datos.

Hechos estos apuntes, concordamos con la doctrina que sostiene la justa retribución y otorga remuneraciones al titular de los datos por un tiempo determinado, según la ley del país de que se trate y sus justas necesidades y realidades.

Finalmente, referente a la celebración de acuerdos internacionales, reafirmamos la idea de que una participación pluralista y en igualdad de condiciones en la mesa de negociaciones en el tratamiento de estos temas a nivel internacional podría evitar futuras inconsistencias en la interpretación de la norma, tal como está ocurriendo con el controvertido régimen de concesión de derechos exclusivos sobre datos de prueba en la actualidad, sobre el cual pretendimos ahondar, esclarecer y aportar.

\section{REFERÊNCIAS}

BASHEER, Shamnad. Protection of Regulatory Data Under Article 39.3 of Trips: The Indian Context. Intelectual Property Institute Journal, 2006. Disponible en: $<$ https://ssrn.com/abstract=934269 $>$. Consultado el 7 de mayo de 2019. 
CHAUDHURI, Sudip. The WTO and India's pharmaceuticals industry. Patent protection, TRIPS and Developing countries. Nueva Delhi: Oxford University Press, 2005.

COMMISSION ON INTELLECTUAL PROPERTY RIGHTS, INNOVATION AND PUBLIC HEALTH (CIPIH). Public Health, Innovation and Intellectual Property Rights, World Health Organization, 2006.2 Disponible en: $<$ http://www.who.int/intellectualproperty/documents/thereport/CIPIH23032006.pdf.> Consultado el 29 de abril y 9 de mayo de 2019.

CORREA, Carlos M. Derechos de Propiedad Intelectual. Competencia y protección del interés público. La nueva ofensiva en materia de observancia de los derechos de propiedad intelectual y los intereses de los países en desarrollo. Buenos Aires: Editora B de F, 2009.

CORREA, Carlos. Protección de datos de prueba: Derechos conferidos bajo el Acuerdo sobre los ADPIC y algunos efectos de los estándares ADPIC-PLUS en Acceso a los medicamentos e información confidencial. Buenos Aires: Ciudad Argentina-Hispania libros, 2014.

. Protection of Data Submitted for the Registration of Pharmaceuticals: Implementing the standards of the TRIPS Agreement. Ginebra: South Centre, Geneva, 2002.

DEPARTAMENTO DE JUSTICIA DE LOS ESTADOS UNIDOS AMERICANOS. Directrices sobre Defensa de la Competencia para la Concesión de Licencias de Propiedad Intelectual del Departamento de Justicia y la Comisión Federal de Comercio del 4/6/1995. Disponible en: <http://www.usdoj.gov/atr/public/guidelines/0558.htm.>. Consultado el 9 de mayo de 2019.

DNDi. Edición en internet: <https://www.dndi.org/>. Consultado el 29 abril de 2019.

FAO. Food and Agriculture Organization of the United Nations (FAO). Panel of Eminent Expets on Ethics in Food and Agriculture. Report of Eminent Experts on Ethics in Food and Agriculture, Third Session, 14-16 September 2005. Disponible en: <http://www.fao.org/docrep/010/a0697e/a0697e00.HTM>. Consultado el 7 de mayo de 2019.

FELLMETH, Aaron. Secrecy, Monopoly, and Acces to Pharmaceuticals in Internacional Trade Law: Protection of Marketing Approval Data under the TRIPS Agreement. Harvard International Law Journal, volumen 45, número 2, 2004. Disponible en: <http://www.harvardilj.org/wp-content/uploads/2011/04/HILJ 452 Fellmeth.pdf>. Consultado el 7 de mayo de 2019. 
FORGIONI, Paula A. Os fundamentos do antitruste. San Pablo: Editora Revista dos Tribunais, 2016.

GIMÉNEZ PEREIRA, Marta. Efectos de la Protección de Las Patentes Farmacéuticas: Un análisis de propiedad intelectual. Ciudad de México: Tirant lo Blanch, 2017.

MEITINGER, Ingo. Implementation of Test Data Protection According to the Article 39.3 TRIPS. The Search for a Fair Interpretation of the Term 'Unfair Commercial Use'. The Journal of World Intellectual Property, volumen 8, número 2, 2005. Disponible en: <https://doi.org/10.1111/j.1747-1796.2005.tb00243.x>. Consultado el 7 de mayo de 2019.

MINISTERIO DE JUSTICIA Y DERECHOS HUMANOS. PRESIDENCIA DE LA NACIÓN. ARGENTINA. InfoLEG. Ley de Patentes argentina. Disponible en: $<$ http://servicios.infoleg.gob.ar/infoleglnternet/anexos/35000-

39999/35001/texact.htm>. Consultada el 9 de mayo de 2019.

OMC. Declaración Ministerial de Doha WT/MIN(01)/DEC/1, 20 de noviembre de $2001 . \quad$ Disponible en: $<$ http://www.wto.org/english/thewto e/minist e/min01 e/mindecl e.htm $>$. Consultada el 15 de abril de 2019.

TRIPS and Public Health, Submission by the African Group, Barbados, Bolivia, Brazil, Dominican Republic, Ecuador, Honduras, India, Indonesia, Jamaica, Pakistan, Paraguay, Philippines, Peru, Sri Lanka, Thailand and Venezuela, IP/C/W/296, 19 de junio de 2001. Disponible en: $<$ http://www.wto.org/english/tratop e/trips e/paper develop w296 e.htm>.

Consultado el 7 mayo de 2019.

Acuerdo de la Ronda Uruguay: ADPIC, Parte II - Normas relativas a la existencia, alcance y ejercicio de los derechos de propiedad intelectual, Sección 7: Protección de la información no divulgada. 1994. Disponible en: $<$ https://www.wto.org/spanish/docs s/legal s/27-trips 04d s.htm>. Consultado el 7 mayo de 2019.

SATWANT, Reddy; GURDIAL, Singh Sandhu. Report on Steps to be taken by Government of India in the context of Data Protection Provisions of Article 39.3 of TRIPS Agreement 0 Informe de India. Disponible en: $<$ https://www.indiaoppi.com/sites/default/files/PDF\%20files/Data\%20Protection\%20re port.PDF>. Consultado el 7 de mayo de 2019.

UE. Directrices sobre la aplicabilidad del artículo 81 del Tratado de la CE a los acuerdos de cooperación horizontal (2001/C 3/02). Disponible en: <https://eurlex.europa.eu/legal- 
content/ES/TXT/?uri=uriserv:OJ.C .2001.003.01.0002.01.SPA\&toc=OJ:C:2001:003: TOC>. Consultado el 9 de mayo de 2019.

Directrices sobre la aplicabilidad del artículo 101 del Tratado de Funcionamiento de la Unión Europea a los acuerdos de cooperación horizontal, Texto pertinente a efectos del EEE. Disponible en: <https://eur-lex.europa.eu/legalcontent/es/AUTO/?uri=CELEX:52011XC0114(04)>. Consultado el 9 de mayo de 2019. 